\title{
Small RNAs in the peripheral blood discriminate metastasized from non-metastasized seminoma
}

\author{
Christian G Ruf ${ }^{1,2^{*}}$, Daniela Dinger ${ }^{1}$, Matthias Port ${ }^{2,4}$, Hans-Ulrich Schmelz ${ }^{2,3}$, Walter Wagner ${ }^{1}$, Cord Matthies ${ }^{1}$, \\ Bertram Müller-Myhsok ${ }^{5}$, Viktor Meineke ${ }^{2}$ and Michael Abend ${ }^{2}$
}

\begin{abstract}
Background: We aimed to better discriminate metastasized (lymphogen/occult/both combined) from non-metastasized seminoma based on post-transcriptional changes examined in the peripheral blood.

Methods: Total RNAs including small RNAs were isolated from the peripheral blood of patients suffering from metastasized testicular tumours (lymphogen, $n=5$, clinical stage $l \mathrm{lb} / \mathrm{c}$; occult, $n=5$, clinical stage $\mathrm{I}$ ) and non-metastasized patients ( $n=5$, clinical stage I). Small RNA next generation sequencing (SOLID, Life Technologies) was employed to examine post-transcriptional changes. We searched for small RNAs showing at least 50 reads and a significant $\geq 2$-fold difference using peripheral blood small RNAs of non-metastasized tumours as the reference group. Candidate small RNAs were examined in univariate logistic regression analysis and combinations of two small RNAs were further examined using support vector machines.
\end{abstract}

Results: On average $1.3 \times 10^{7}, 1.2 \times 10^{7}$ and $1.2 \times 10^{7}$ small RNA reads were detectable in non-metastasized, lymphogen and occult metastasized seminoma, respectively of which $73-76 \%$ remained after trimming. From these between

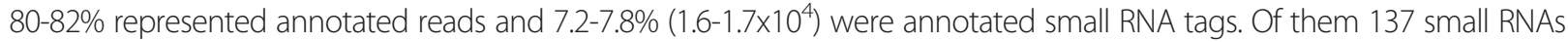
showed $>50$ reads and a $\geq$ two-fold difference to the reference. In univariate analysis we detected 33-35 different small RNAs which significantly discriminated lymphogen/occult/combined metastasized from non-metastasized seminoma and among these different comparisons it were the same small RNAs in 44-79\%. Many combinations of two of these small RNAs completely discriminated metastasized from non-metastasized seminoma irrespective of the metastasis subtype.

Conclusions: Metastasized (either lymphogen or occult) seminoma can be completely discriminated from non-metastasized seminoma with a combination of two small RNAs measured in the peripheral blood.

Keywords: Testis tumour, Gene expression, Small RNA, MicroRNA, Metastasized seminoma, Next generation sequencing, Risk factor, Tumour marker, Blood

\section{Background}

Testicular tumor, as the most common tumor in young men, is associated with a 5 year survival rate close to $100 \%$ in early stages. Pure seminoma are the most frequent histological subtype (55\%) nowadays and more than $70 \%$ of patients present without visible metastasis at primary staging [1]. Gold standard for primary staging is computed tomography $(\mathrm{CT})$ of the chest, abdomen and pelvis to detect metastases. In truly non-metastasized clinical stage I (cS I) patients are cured by orchidectomy

\footnotetext{
* Correspondence: Dr.ChristianRuf@gmx.de

'Department of Urology, Federal Armed Forces Hospital, 22049 Hamburg, Germany

${ }^{2}$ Bundeswehr Institute of Radiobiology, 80937 Munich, Germany

Full list of author information is available at the end of the article
}

alone, but despite modern staging and classification procedures up to $30 \%$ of cS I seminoma patients' bear occult metastasis in primary staging and relapse after orchidectomy alone $[2,3]$. Until today no reliable biological parameters exist and clinical parameter are showing a concordance of $65 \%$ only in differentiating occult metastasized stages from non-metastasized seminoma [4]. Identification of occult metastasized patients is one of the main goals to prevent toxicity (e.g. cardiovascular and kidney disease, secondary malignancies and decreased fertility) caused by unnecessary adjuvant treatment or diagnostic procedures (additional radiation exposure due to quarterly CT scans) during follow up [5]. 
Recently, other authors started to examine whether a certain set of micro RNAs (miRNA) might be suitable for discriminating between seminoma bearing patients and healthy persons [6-8]. Expression of miRNAs in testicular germ cell cancer is also known to be associated with the histologic subtype [7] as well as cisplatin resistance $[9,10]$. Additionally miRNAs are known to be involved in different processes of metastatic spread in other tumours [11]. Among seminoma bearing patients circulating tumour cells are already detected in the peripheral blood [12]. We wondered whether changes in microRNA expression in the peripheral blood might be able to discriminate metastasized (either lymphogen, occult or a combination of both subtypes) from non-metastasized seminoma. We utilized an agnostic approach investigating the whole genome for any kind of small RNA species suitable to discriminate metastatic stage in seminoma employing next generation sequencing (NGS) on peripheral blood samples drawn at the time of the primary tumour's diagnosis.

\section{Results}

\section{Characteristics of seminoma groups}

The average age at diagnosis was $39.1(+/-7.2)$ years for non-metastasized, higher (45.2 years, +/- 10.8) for lymphogen metastasized and lower (32.1 years, $+/-5.4$ ) for occult metastasized seminoma. Primary tumor size was comparable between lymphogen and occult metastasized seminoma ( $37.8 \mathrm{~mm}$ and $38.6 \mathrm{~mm}$, respectively), but smaller $(23.8 \mathrm{~mm})$ in non-metastasized seminoma (Table 1).

\section{RNA isolation}

Per $2.5 \mathrm{ml}$ whole blood we isolated about the same amount (mean) of total RNA from seminoma patients without metastasis $(7.7 \mu \mathrm{g},+/-1.9)$, lymphogen metastasis $(4.9 \mu \mathrm{g},+/-2.0 \mu \mathrm{g})$ and occult metastasis $(6.8 \mu \mathrm{g},+/-2.4$, Table 1). The RNA integrity number (RIN) ranged between 7.0-8.6 per group. No DNA contamination could be detected in our samples.

\section{Analysis of small RNA next generation sequencing results} The average total number of reads for lymphogen/occult metastasized and non-metastasized seminoma was $1.3 \times 10^{7}, 1.2 \times 10^{7}$ and $1.2 \times 10^{7}$ and on average $73-76 \%$ remained for further analysis after trimming of the reads (Table 2). From these reads $80-84 \%$ appeared annotated reads with $7.2-7.8 \%\left(1.6-1.7 \times 10^{4}\right)$ representing annotated small RNAs. Of them 137 small RNAs showed $>50$ reads and a two-fold difference to the reference. In univariate analysis we identified $33 / 34$ and 35 small RNA species which significantly discriminated lymphogen/occult metastasized and both metastasis subtypes combined from non-metastasized seminoma, respectively (Table 3 ). The overlap of small RNAs separating either lymphogen or occult metastasis from non-metastasized seminoma was $47-48 \%$ and increased up to $79 \%$ when comparing lymphogen and occult small RNAs candidates with the small RNAs based on the combined metastasis subtypes (Figure 1). We finally employed support vector machines which completely separated lymphogen, occult metastasis and the combined metastasis subtypes from non-metastasized seminoma using a combination of two small RNA species only (Table 4). Altogether, 891, 668 and 87 combinations allowed a complete separation of lymphogen $(n=5)$, occult metastasis $(n=5)$ and the combined metastasis subtypes $(n=10)$ from non-metastasized seminoma $(n=5)$, respectively.

\section{Discussion}

In our study we aimed to better discriminate metastasized (either lymphogen or occult subtypes or both combined) from non-metastasized seminoma based on small RNA changes examined in the peripheral blood. A whole genome screening on small RNA species was performed employing NGS. We demonstrated each metastasis subtype and its combination to be completely discriminated from non-metastasized seminoma using two small RNAs combined.

MicroRNAs are previously described to be involved in different distant (e.g. migration, angiogenesis and colonization of distant organs) and local (e.g. changes in tumor microenvironment) processes in metastatic cascade. Interestingly, the microRNAs identified in our study are involved in local tumour microenvironmental changes only. For instance, members of the Let-7 family are involved in inflammatory processes as a part of the metastatic cascade. The Let-7 family targets oncogenes such as HMGA2 and KRAS and seem to be a key component in a so called epigenetic switch [13-15]. Cancer associated fibroblasts are involved in tumor formation and progression where miR-15 and miR-16 regulate FGF2 and FGFR1 in prostate cancer [16] and miR-18 in breast cancer [17].

Recently other authors demonstrated that microRNA 371-73 cluster and microRNA 302 discriminated seminoma bearing patients from healthy persons [6-8]. We did the next step and examined the suitability of these microRNA to predict the metastasis status, these microRNA 371-73 cluster and miRNA 302 was detectable in our analysis, but appeared not significantly associated with the metastasis status as it is true for protein tumour markers (e.g. HCG) as well.

The complete separation of metastasis from nonmetastasized seminoma using a combination of two small RNA species points to the significant diagnostic potential of these biological marker which is in line with previous examinations on the transcriptional level showing the superiority of molecular marker over epidemiological or clinical-histological parameter [18,19]. After 
Table 1 Characteristics of patients, their biopsies and RNA isolates

\begin{tabular}{|c|c|c|c|c|c|c|c|c|c|c|}
\hline$\#$ & $\begin{array}{l}\text { Metastasis detection at time of } \\
\text { primary tumor's diagnosis }\end{array}$ & $\begin{array}{c}\text { Age at } \\
\text { diagnosis (years) }\end{array}$ & $\begin{array}{l}\text { Tumor } \\
\text { size }(\mathrm{mm})\end{array}$ & $\mathrm{pL}$ & $\mathrm{pV}$ & pT & $\begin{array}{l}\text { Infiltration } \\
\text { rete testis }\end{array}$ & Initial clinical stage & Total RNA $(\mu \mathrm{g})$ & RIN \\
\hline 1 & Non metastasized & 38 & 14 & 0 & 0 & 1 & $n$ & $\mathrm{cSI}$ & 7.6 & 7.8 \\
\hline 2 & & 50 & 22 & 0 & 0 & 1 & $\mathrm{n}$ & $\mathrm{cSI}$ & 8.6 & 8.3 \\
\hline 3 & & 31 & 19 & 1 & 0 & 1 & $\mathrm{n}$ & cSI & 6.4 & 7.0 \\
\hline 4 & & 42 & 45 & 0 & 0 & 1 & y & cSI & 3.9 & 8.0 \\
\hline 5 & & 35 & 19 & 0 & 0 & 1 & y & $\mathrm{cSI}$ & 8.2 & 7.6 \\
\hline Mean & & 39.1 & 23.8 & & & & & & 6.9 & 7.7 \\
\hline stdev & & 7.2 & 12.2 & & & & & & 1.9 & 0.5 \\
\hline 1 & Lymphogen metastasized & 32 & 40 & 0 & 0 & 1 & $n$ & cSllb & 5.0 & 8.5 \\
\hline 2 & & 50 & 12 & 0 & 0 & 3 & $n$ & cSllc & 5.1 & 8.8 \\
\hline 3 & & 43 & 50 & 0 & 0 & 1 & $n$ & cSllb & 8.1 & 8.3 \\
\hline 4 & & 42 & 45 & 1 & 0 & 2 & y & cSllb & 3.2 & 9.3 \\
\hline 5 & & 61 & 42 & 0 & 0 & 1 & y & cSllc & 3.2 & 8.2 \\
\hline Mean & & 45.2 & 37.8 & & & & & & 4.9 & 8.6 \\
\hline stdev & & 10.8 & 14.9 & & & & & & 2.0 & 0.4 \\
\hline 1 & Occult metastasized & 33 & 55 & 1 & 0 & 2 & $n$ & cSI & 5.0 & 6.0 \\
\hline 2 & & 37 & 35 & 0 & 0 & 1 & y & cSI & 4.1 & 7.4 \\
\hline 3 & & 37 & 30 & 0 & 0 & 1 & $n$ & CSI & 7.0 & 7.8 \\
\hline 4 & & 31 & 18 & 0 & 0 & 1 & $n$ & $\mathrm{cSI}$ & 8.1 & 7.4 \\
\hline 5 & & 23 & 55 & 0 & 0 & 1 & $\mathrm{n}$ & cSI & 10.0 & 6.4 \\
\hline Mean & & 32.1 & 38.6 & & & & & & 6.8 & 7.0 \\
\hline stdev & & 5.4 & 16.2 & & & & & & 2.4 & 0.8 \\
\hline
\end{tabular}

Table 2 Descriptive statistics of number of reads before and after trimming and the percentage of annotated small RNAs measured in the peripheral blood of patients suffering from seminoma without metastasis as well as lymphogen and occult metastasized seminoma

\begin{tabular}{|c|c|c|c|c|c|c|c|c|}
\hline \multirow{2}{*}{$\begin{array}{c}\text { Seminoma } \\
\text { metastasis status }\end{array}$} & \multirow{2}{*}{$\begin{array}{l}\text { Total no. } \\
\text { of reads }\end{array}$} & \multicolumn{2}{|c|}{ Reads after trimming } & \multicolumn{2}{|c|}{ Annotated reads } & \multicolumn{3}{|c|}{ Small RNA tags } \\
\hline & & abs. & $\%$ & abs. & $\%$ & abs. & abs. annotated & $\%$ \\
\hline \multicolumn{9}{|c|}{ Non-metastasized, $n=5$} \\
\hline Mean & $1.3 E+07$ & $9.7 \mathrm{E}+06$ & 76 & $8.1 \mathrm{E}+06$ & 84 & $2.2 \mathrm{E}+05$ & $1.6 \mathrm{E}+04$ & 7.2 \\
\hline Stdev & $2.0 \mathrm{E}+06$ & $1.6 \mathrm{E}+06$ & 2.2 & $1.4 \mathrm{E}+06$ & 2.2 & $5.4 \mathrm{E}+04$ & $3.4 \mathrm{E}+03$ & 0.7 \\
\hline Min & $9.4 \mathrm{E}+06$ & $7.1 \mathrm{E}+06$ & 72 & $5.7 \mathrm{E}+06$ & 81 & $1.7 \mathrm{E}+05$ & $1.2 \mathrm{E}+04$ & 6.5 \\
\hline Max & $1.4 \mathrm{E}+07$ & $1.1 \mathrm{E}+07$ & 78 & $9.5 \mathrm{E}+06$ & 85 & $3.1 \mathrm{E}+05$ & $2.0 \mathrm{E}+04$ & 8.2 \\
\hline \multicolumn{9}{|c|}{ Lymphogen metastasized, $n=5$} \\
\hline Mean & $1.2 \mathrm{E}+07$ & $7.8 \mathrm{E}+06$ & 73 & $6.3 \mathrm{E}+06$ & 80 & $2.3 \mathrm{E}+05$ & $1.6 \mathrm{E}+04$ & 7.3 \\
\hline Stdev & $6.5 E+06$ & $3.1 \mathrm{E}+06$ & 16 & $2.7 E+06$ & 3.8 & $8.6 \mathrm{E}+04$ & $3.2 \mathrm{E}+03$ & 1.5 \\
\hline Min & $5.7 \mathrm{E}+06$ & $4.8 \mathrm{E}+06$ & 45 & $3.6 \mathrm{E}+06$ & 75 & $1.5 \mathrm{E}+05$ & $1.3 \mathrm{E}+04$ & 5.6 \\
\hline Max & $2.0 \mathrm{E}+07$ & $1.2 \mathrm{E}+07$ & 86 & $1.0 \mathrm{E}+07$ & 85 & $3.3 \mathrm{E}+05$ & $1.9 \mathrm{E}+04$ & 8.5 \\
\hline \multicolumn{9}{|c|}{ Occult metastasized, $n=5$} \\
\hline Mean & $1.2 \mathrm{E}+07$ & $9.0 \mathrm{E}+06$ & 73 & $7.3 E+06$ & 82 & $2.3 \mathrm{E}+05$ & $1.7 \mathrm{E}+04$ & 7.8 \\
\hline Stdev & $4.3 \mathrm{E}+06$ & $3.5 \mathrm{E}+06$ & 6.6 & $2.8 \mathrm{E}+06$ & 2.5 & $5.1 \mathrm{E}+04$ & $9.4 \mathrm{E}+02$ & 1.4 \\
\hline Min & $6.7 \mathrm{E}+06$ & $4.7 \mathrm{E}+06$ & 64 & $3.8 \mathrm{E}+06$ & 79 & $1.8 \mathrm{E}+05$ & $1.6 \mathrm{E}+04$ & 5.9 \\
\hline Max & $1.9 \mathrm{E}+07$ & $1.4 \mathrm{E}+07$ & 81 & $1.1 \mathrm{E}+07$ & 85 & $3.1 E+05$ & $1.8 \mathrm{E}+04$ & 10 \\
\hline
\end{tabular}


Table 3 Summary of significantly associated small RNAs with metastasis status per group using logistic regression analysis (univariate)

\begin{tabular}{|c|c|c|c|c|c|c|c|c|c|c|c|c|c|c|}
\hline \multicolumn{5}{|c|}{ Non versus metastasized (lymphogen and occult, $n=35$ ) } & \multicolumn{5}{|c|}{ Non versus lymphogen metastasized $(n=33)$} & \multicolumn{5}{|c|}{ Non versus occult metastasized $(n=34)$} \\
\hline \multirow{2}{*}{$\begin{array}{l}\text { Small RNA } \\
\text { let-7f-1 }\end{array}$} & \multirow{2}{*}{$\begin{array}{l}\mathbf{p} \text {-value } \\
0.04\end{array}$} & \multirow{2}{*}{$\begin{array}{l}\text { Fold-change } \\
5.5\end{array}$} & \multicolumn{2}{|c|}{$95 \%$ confidence interval } & \multirow{2}{*}{$\begin{array}{l}\text { Small RNA } \\
\text { let-7f-1 }\end{array}$} & \multirow{2}{*}{$\begin{array}{l}\mathbf{p} \text {-value } \\
0.03\end{array}$} & \multirow{2}{*}{$\begin{array}{l}\text { Fold-change } \\
7.2\end{array}$} & \multicolumn{2}{|c|}{$\begin{array}{l}95 \% \text { confidence } \\
\text { interval }\end{array}$} & \multirow{2}{*}{$\begin{array}{l}\text { Small RNA } \\
\text { let-7a-1 }\end{array}$} & \multirow{2}{*}{$\begin{array}{l}\mathbf{p} \text {-value } \\
0.02\end{array}$} & \multirow{2}{*}{$\begin{array}{l}\text { Fold-change } \\
3.1\end{array}$} & \multicolumn{2}{|c|}{$\begin{array}{l}95 \% \text { confidence } \\
\text { interval }\end{array}$} \\
\hline & & & 1.3 & 23.7 & & & & 1.6 & 32.4 & & & & 1.4 & 7.0 \\
\hline mir-16-1 & 0.05 & 2.2 & 1.1 & 4.5 & let-7f-2 & 0.04 & 8.1 & 1.5 & 44.6 & let-7a-2 & 0.02 & 3.1 & 1.4 & 7.1 \\
\hline mir-18a & 0.05 & 2.4 & 1.1 & 5.2 & mir-15a & 0.05 & 5.0 & 1.3 & 19.4 & let-7a-3 & 0.02 & 3.1 & 1.4 & 7.0 \\
\hline mir-23a & 0.05 & 2.7 & 1.1 & 6.5 & mir-16-1 & 0.001 & 3.2 & 2.1 & 5.0 & let-7b & 0.01 & 3.1 & 1.6 & 6.1 \\
\hline mir-92a-1 & 0.008 & 2.7 & 1.4 & 5.1 & mir-92a-1 & 0.007 & 3.0 & 1.6 & 5.5 & let-7d & 0.006 & 2.7 & 1.6 & 4.6 \\
\hline mir-92a-2 & 0.008 & 2.8 & 1.5 & 5.2 & mir-92a-2 & 0.007 & 3.0 & 1.6 & 5.6 & let-7f-1 & 0.006 & 3.8 & 1.9 & 7.6 \\
\hline mir-99a & 0.01 & 2.5 & 1.3 & 4.6 & mir-183 & 0.001 & 2.5 & 1.7 & 3.6 & let-7f-2 & 0.01 & 3.8 & 1.7 & 8.3 \\
\hline mir-183 & 0.02 & 2.0 & 1.2 & 3.5 & let-7 g & 0.03 & 6.2 & 1.6 & 23.0 & mir-18a & 0.04 & 2.3 & 1.2 & 4.5 \\
\hline let-7 g & 0.05 & 4.5 & 1.2 & 17.6 & mir-15b & 0.02 & 6.8 & 1.8 & 26.2 & mir-92a-1 & 0.03 & 2.5 & 1.3 & 4.8 \\
\hline mir-23b & 0.04 & 2.8 & 1.1 & 7.1 & mir-23b & 0.04 & 3.3 & 1.3 & 8.3 & mir-92a-2 & 0.03 & 2.5 & 1.3 & 4.8 \\
\hline mir-130a & 0.001 & 0.2 & 0.1 & 0.4 & mir-130a & 0.04 & 0.2 & 0.1 & 0.7 & mir-99a & 0.006 & 2.5 & 1.5 & 4.0 \\
\hline mir-191 & 0.004 & 0.4 & 0.2 & 0.7 & mir-142 & 0.03 & 0.5 & 0.3 & 0.8 & let-7 g & 0.03 & 2.9 & 1.3 & 6.4 \\
\hline mir-296 & 0.01 & 2.3 & 1.3 & 4.0 & mir-191 & 0.03 & 0.4 & 0.2 & 0.8 & mir-30b & 0.03 & 2.2 & 1.2 & 3.8 \\
\hline mir-378a & 0.01 & 2.4 & 1.3 & 4.3 & mir-126 & 0.03 & 2.0 & 1.2 & 3.4 & mir-130a & 0.03 & 0.1 & 0.0 & 0.5 \\
\hline mir-326 & 0.02 & 2.7 & 1.3 & 5.5 & mir-296 & 0.01 & 2.2 & 1.4 & 3.6 & mir-191 & 0.01 & 0.4 & 0.2 & 0.7 \\
\hline mir-331 & 0.01 & 3.0 & 1.4 & 6.3 & mir-331 & 0.02 & 2.7 & 1.4 & 5.3 & mir-29c & 0.04 & 2.1 & 1.1 & 3.8 \\
\hline mir-339 & 0.04 & 2.1 & 1.1 & 4.0 & mir-425 & 0.05 & 3.7 & 1.2 & 11.0 & mir-296 & 0.04 & 2.4 & 1.2 & 4.7 \\
\hline mir-425 & 0.01 & 3.9 & 1.6 & 9.4 & mir-451a & 0.009 & 2.2 & 1.4 & 3.4 & mir-378a & 0.006 & 2.5 & 1.5 & 4.2 \\
\hline mir-451a & 0.002 & 2.2 & 1.5 & 3.2 & mir-92b & 0.02 & 2.2 & 1.3 & 3.5 & mir-326 & 0.01 & 3.4 & 1.7 & 6.9 \\
\hline mir-92b & 0.02 & 2.5 & 1.2 & 5.0 & mir-574 & 0.005 & 0.3 & 0.2 & 0.6 & mir-331 & 0.03 & 3.3 & 1.4 & 7.9 \\
\hline mir-574 & 0.0001 & 0.4 & 0.2 & 0.5 & mir-4286 & 0.04 & 3.3 & 1.3 & 8.7 & mir-339 & 0.02 & 2.6 & 1.4 & 4.9 \\
\hline mir-4286 & 0.02 & 3.9 & 1.5 & 10.5 & mir-4454 & 0.008 & 2.3 & 1.4 & 3.7 & mir-425 & 0.002 & 4.1 & 2.2 & 7.5 \\
\hline mir-4454 & 0.03 & 2.7 & 1.2 & 5.9 & ENST00000516594 & 0.007 & 0.4 & 0.2 & 0.6 & mir-451a & 0.01 & 2.2 & 1.4 & 3.5 \\
\hline ENST00000516594 & 0.02 & 0.5 & 0.3 & 0.8 & ENST00000363271 & 0.007 & 0.4 & 0.2 & 0.6 & mir-92b & 0.04 & 2.8 & 1.2 & 6.4 \\
\hline ENST00000365160 & 0.03 & 0.4 & 0.2 & 0.8 & ENST00000459091 & 0.002 & 0.3 & 0.2 & 0.5 & mir-574 & 0.0009 & 0.4 & 0.3 & 0.6 \\
\hline ENST00000363271 & 0.02 & 0.5 & 0.3 & 0.8 & ENST00000516350 & 0.007 & 0.4 & 0.2 & 0.6 & mir-660 & 0.03 & 2.4 & 1.3 & 4.5 \\
\hline ENST000000459091 & 0.006 & 0.4 & 0.3 & 0.7 & ENST00000517209 & 0.002 & 0.4 & 0.2 & 0.6 & mir-664 & 0.02 & 2.1 & 1.3 & 3.5 \\
\hline ENST00000516350 & 0.02 & 0.5 & 0.3 & 0.8 & ENST00000516507 & 0.007 & 0.4 & 0.2 & 0.7 & mir-4286 & 0.02 & 4.5 & 1.7 & 11.8 \\
\hline ENST00000363865 & 0.02 & 0.5 & 0.3 & 0.8 & ENST00000363865 & 0.007 & 0.4 & 0.2 & 0.6 & mir-4454 & 0.05 & 3.0 & 1.2 & 7.5 \\
\hline ENST00000362808 & 0.02 & 0.5 & 0.3 & 0.8 & ENST00000362808 & 0.007 & 0.4 & 0.2 & 0.6 & ENST00000365160 & 0.04 & 0.3 & 0.1 & 0.8 \\
\hline
\end{tabular}


Table 3 Summary of significantly associated small RNAs with metastasis status per group using logistic regression analysis (univariate) (Continued)

\begin{tabular}{|c|c|c|c|c|c|c|c|c|c|c|c|c|c|c|}
\hline ENST00000364409 & 0.02 & 0.5 & 0.3 & 0.8 & ENST00000364409 & 0.007 & 0.4 & 0.2 & 0.6 & ENST00000387347 & 0.01 & 3.1 & 1.6 & 6.0 \\
\hline ENST00000363745 & 0.02 & 0.5 & 0.3 & 0.8 & ENST00000363745 & 0.007 & 0.4 & 0.2 & 0.6 & ENST00000482884 & 0.05 & 2.6 & 1.2 & 5.6 \\
\hline ENST00000387347 & 0.03 & 3.0 & 1.3 & 7.3 & ENST00000461337 & 0.007 & 0.4 & 0.2 & 0.6 & ENST00000459949 & 0.002 & 2.3 & 1.6 & 3.4 \\
\hline ENST00000459949 & 0.003 & 2.0 & 1.4 & 2.9 & & & & & & ENST00000410361 & 0.02 & 3.3 & 1.4 & 7.8 \\
\hline ENST00000461337 & 0.02 & 0.5 & 0.3 & 0.8 & & & & & & & & & & \\
\hline
\end{tabular}




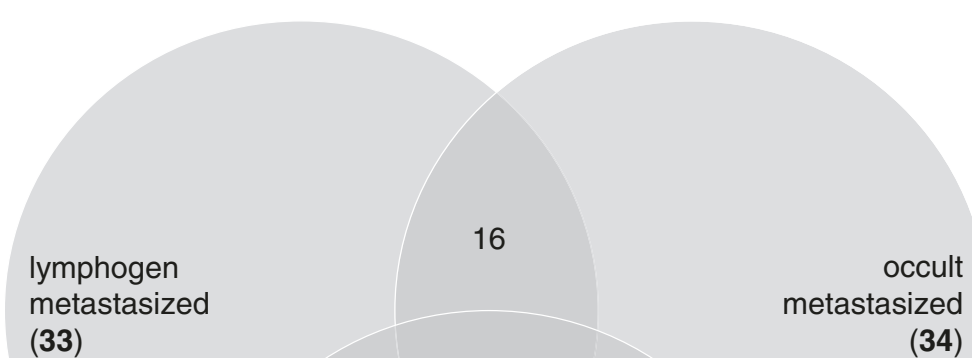

15

26

21

metastasized,

both (35)

Figure 1 Venn diagram showing the total number (bold) and overlapping number of significantly associated small RNAs (from Table 3) to discriminate different metastasis subtypes (lymphogen, occult and the combination of both subtypes) from non-metastasized seminoma.

validation of these results on a larger group, these findings could help for therapy decision making in favour of adjuvant chemotherapy or surveillance based on miRNA expression changes predicting metastasis spread, even if metastasis spreads are not visible using a CT scan.

Interesting, the discrimination in our study occurred irrespective of the metastasis subtype and with the same microRNAs in up to $79 \%$. This was expected, since we recently demonstrated lymphogen and occult metastasized seminoma to be indistinguishable on the transcriptional and post-transcriptional level (revised version submitted).

Noteworthy, these changes were examined in the peripheral blood of seminoma patients taken at the time of the primary tumour's diagnosis. It can be hypothesized that our measurements are linked to seminoma tumour cells circulating in the peripheral blood.

Our study has certain weaknesses such as the low number of cases examined (total $\mathrm{n}=15$ ). However, the molecular biological methodology applied provides a deepness, which under financial considerations does not allow large scale studies. Hence, this study provides hints towards certain small RNA species comprising a significant diagnostic potential for prediction of seminoma metastasis based on examinations in the peripheral blood, but certainly these candidate small RNA species have to be examined on a larger independent group for validation purposes. The validation should also include haematogenous metastasized seminoma (clinical Stage III), not included in this study, to prove the usability in this tumor stage as well. Furthermore, we did not adjust the p-values for multiple comparisons, because of the explorative character of this study and the low sample size. Based on experiences with previous work we expect about $10-20 \%$ of our candidates being false positives/negatives [20]. However, this study represents a screening approach. About 100 most promising candidate genes will be considered for validation in a following study using another group and another methodology (qRT-PCR).

\section{Conclusion}

Metastasized (either lymphogen or occult) seminoma can be completely discriminated from non-metastasized seminoma with a combination of two small RNAs measured in the peripheral blood.

\section{Methods}

\section{Patient selection}

Non-metastasized seminoma $(n=5)$ received no adjuvant treatment and were free of relapse/progress for at least two years of follow up. Occult metastasized patients $(n=$ 5) presented without visible metastasis at primary staging, received no adjuvant treatment, and developed retroperitoneal tumour progress during the follow up of 12 month. For patients with detectable metastasis at primary staging $(n=5)$ we focused on clinical stage IIb and IIc, to include lymphogenic metastatic spread only and to provide a high level of diagnostic accuracy (avoiding doubtful lymph 
Table 4 Summary on combinations of two small RNAs (small RNA1 with small RNA2) which together completely discriminate metastasis subtypes (lymphogen, occult and their combination) from non-metastasized seminoma employing support vector machines

\begin{tabular}{|c|c|c|c|c|c|}
\hline \multicolumn{2}{|c|}{$\begin{array}{l}\text { Non versus metastasized } \\
\text { (lymphogen and occult, } n=87 \text { ) }\end{array}$} & \multicolumn{2}{|c|}{$\begin{array}{l}\text { Non versus lymphogen metastasized } \\
(n=87 \text { from 891) }\end{array}$} & \multicolumn{2}{|c|}{$\begin{array}{l}\text { Non versus occult metastasized } \\
(=87 \text { from } 668)\end{array}$} \\
\hline Small RNA1 & Small RNA2 & Small RNA1 & Small RNA2 & Small RNA1 & Small RNA2 \\
\hline let-7f-1 & mir-191 & let-7a-1 & mir-192 & let-7a-1 & mir-223 \\
\hline let-7f-1 & mir-574 & let-7a-1 & mir-191 & let-7a-1 & mir-574 \\
\hline let-7f-2 & mir-191 & let-7a-1 & mir-532 & let-7a-1 & mir-4454 \\
\hline mir-18a & mir-532 & let-7a-1 & ENST00000516594 & let-7a-2 & mir-223 \\
\hline mir-19b-1 & mir-342 & let-7a-1 & ENST00000363271 & let-7a-2 & mir-574 \\
\hline mir-19b-1 & ENST00000517209 & let-7a-1 & ENST00000459091 & let-7a-2 & mir-4454 \\
\hline mir-19b-2 & mir-342 & let-7a-1 & ENST00000516350 & let-7a-3 & mir-223 \\
\hline mir-19b-2 & ENST00000517209 & let-7a-1 & ENST00000517209 & let-7a-3 & mir-574 \\
\hline mir-28 & mir-574 & let-7a-1 & ENST00000516501 & let-7a-3 & mir-4454 \\
\hline mir-29a & mir-223 & let-7a-1 & ENST00000516507 & let-7b & mir-16-1 \\
\hline mir-29a & mir-574 & let-7a-1 & ENST00000363865 & let-7b & mir-19b-1 \\
\hline mir-93 & mir-532 & let-7a-1 & ENST00000362808 & let-7b & mir-19b-2 \\
\hline mir-99a & mir-574 & let-7a-1 & ENST00000364409 & let-7b & mir-22 \\
\hline mir-192 & ENST00000459091 & let-7a-1 & ENST00000363745 & let-7b & mir-23a \\
\hline mir-197 & mir-92b & let-7a-1 & ENST00000461337 & let-7b & mir-24-1 \\
\hline mir-197 & mir-4454 & let-7a-2 & mir-192 & let-7b & mir-24-2 \\
\hline mir-183 & mir-191 & let-7a-2 & mir-191 & let-7b & $\operatorname{mir}-25$ \\
\hline mir-223 & mir-145 & let-7a-2 & mir-532 & let-7b & mir-26a-1 \\
\hline mir-223 & mir-423 & let-7a-2 & ENST00000516594 & let-7b & mir-26b \\
\hline mir-223 & mir-664 & let-7a-2 & ENST00000363271 & let-7b & mir-28 \\
\hline mir-223 & ENST00000459091 & let-7a-2 & ENST00000459091 & let-7b & mir-29a \\
\hline mir-30b & mir-574 & let-7a-2 & ENST00000516350 & let-7b & mir-29b-1 \\
\hline mir-125b-1 & mir-130a & let-7a-2 & ENST00000517209 & let-7b & mir-29b-2 \\
\hline mir-130a & mir-142 & let-7a-2 & ENST00000516501 & let-7b & mir-103a-2 \\
\hline mir-130a & mir-125b-2 & let-7a-2 & ENST00000516507 & let-7b & mir-103a-1 \\
\hline mir-130a & mir-150 & let-7a-2 & ENST00000363865 & let-7b & mir-107 \\
\hline mir-130a & mir-186 & let-7a-2 & ENST00000362808 & let-7b & mir-16-2 \\
\hline mir-130a & mir-361 & let-7a-2 & ENST00000364409 & let-7b & mir-192 \\
\hline mir-130a & mir-340 & let-7a-2 & ENST00000363745 & let-7b & mir-197 \\
\hline mir-130a & mir-342 & let-7a-2 & ENST00000461337 & let-7b & mir-181a-2 \\
\hline mir-130a & mir-574 & let-7a-3 & mir-192 & let-7b & mir-182 \\
\hline mir-140 & mir-342 & let-7a-3 & mir-191 & let-7b & mir-181a-1 \\
\hline mir-140 & mir-532 & let-7a-3 & mir-532 & let-7b & mir-221 \\
\hline mir-142 & mir-326 & let-7a-3 & ENST00000516594 & let-7b & mir-223 \\
\hline mir-142 & mir-423 & let-7a-3 & ENST00000363271 & let-7b & mir-23b \\
\hline mir-142 & mir-425 & let-7a-3 & ENST00000459091 & let-7b & mir-125b-1 \\
\hline mir-142 & mir-451a & let-7a-3 & ENST00000516350 & let-7b & mir-130a \\
\hline mir-191 & mir-92b & let-7a-3 & ENST00000517209 & let-7b & mir-140 \\
\hline mir-29c & mir-342 & let-7a-3 & ENST00000516501 & let-7b & mir-142 \\
\hline mir-29c & mir-574 & let-7a-3 & ENST00000516507 & let-7b & mir-191 \\
\hline mir-378a & mir-532 & let-7a-3 & ENST00000363865 & let-7b & $\operatorname{mir}-125 a$ \\
\hline
\end{tabular}


Table 4 Summary on combinations of two small RNAs (small RNA1 with small RNA2) which together completely discriminate metastasis subtypes (lymphogen, occult and their combination) from non-metastasized seminoma employing support vector machines (Continued)

\begin{tabular}{|c|c|c|c|c|c|}
\hline mir-378a & mir-574 & let-7a-3 & ENST00000362808 & let-7b & mir-125b-2 \\
\hline mir-342 & mir-423 & let-7a-3 & ENST00000364409 & let-7b & mir-126 \\
\hline mir-342 & mir-425 & let-7a-3 & ENST00000363745 & let-7b & mir-186 \\
\hline mir-342 & mir-3676 & let-7a-3 & ENST00000461337 & let-7b & mir-320a \\
\hline mir-342 & ENST00000387347 & let-7b & mir-192 & let-7b & mir-106b \\
\hline mir-342 & ENST00000482884 & let-7b & mir-191 & let-7b & mir-29c \\
\hline mir-326 & mir-532 & let-7b & mir-532 & let-7b & mir-296 \\
\hline mir-326 & mir-574 & let-7b & ENST00000516594 & let-7b & mir-26a-2 \\
\hline mir-326 & ENST00000517209 & let-7b & ENST00000363271 & let-7b & mir-361 \\
\hline mir-423 & mir-574 & let-7b & ENST00000459091 & let-7b & mir-378a \\
\hline mir-425 & mir-532 & let-7b & ENST00000516350 & let-7b & mir-340 \\
\hline mir-425 & mir-629 & let-7b & ENST00000517209 & let-7b & mir-328 \\
\hline mir-425 & ENST00000517209 & let-7b & ENST00000516501 & let-7b & mir-342 \\
\hline mir-451a & mir-574 & let-7b & ENST00000516507 & let-7b & mir-326 \\
\hline mir-484 & mir-574 & let-7b & ENST00000363865 & let-7b & mir-151a \\
\hline mir-532 & mir-92b & let-7b & ENST00000362808 & let-7b & mir-331 \\
\hline mir-532 & mir-625 & let-7b & ENST00000364409 & let-7b & mir-324 \\
\hline mir-532 & mir-660 & let-7b & ENST00000363745 & let-7b & mir-339 \\
\hline mir-532 & ENST00000482884 & let-7b & ENST00000461337 & let-7b & mir-345 \\
\hline mir-92b & mir-574 & let-7d & mir-192 & let-7b & mir-423 \\
\hline mir-574 & mir-625 & let-7d & mir-191 & let-7b & mir-425 \\
\hline mir-574 & mir-454 & let-7d & mir-532 & let-7b & mir-451a \\
\hline mir-574 & mir-664 & let-7d & ENST00000516594 & let-7b & mir-484 \\
\hline mir-574 & mir-4454 & let-7d & ENST00000363271 & let-7b & mir-505 \\
\hline mir-574 & ENST00000516594 & let-7d & ENST00000459091 & let-7b & mir-532 \\
\hline mir-574 & ENST00000363271 & let-7d & ENST00000516350 & let-7b & mir-574 \\
\hline mir-574 & ENST00000459091 & let-7d & ENST00000517209 & let-7b & mir-652 \\
\hline mir-574 & ENST00000516350 & let-7d & ENST00000516501 & let-7b & mir-766 \\
\hline mir-574 & ENST00000516507 & let-7d & ENST00000516507 & let-7b & mir-744 \\
\hline mir-574 & ENST00000363865 & let-7d & ENST00000363865 & let-7b & mir-1260a \\
\hline mir-574 & ENST00000362808 & let-7d & ENST00000362808 & let-7b & mir-1280 \\
\hline mir-574 & ENST00000364409 & let-7d & ENST00000364409 & let-7b & mir-1260b \\
\hline mir-574 & ENST00000363745 & let-7d & ENST00000363745 & let-7b & mir-4286 \\
\hline mir-574 & ENST00000387347 & let-7d & ENST00000461337 & let-7b & mir-3676 \\
\hline mir-574 & ENST00000459949 & let-7f-1 & mir-192 & let-7b & mir-4454 \\
\hline mir-574 & ENST00000461337 & let-7f-1 & mir-191 & let-7b & ENST00000516572 \\
\hline mir-1260a & ENST00000459091 & let-7f-1 & mir-532 & let-7b & ENST00000516351 \\
\hline mir-3676 & ENST00000517209 & let-7f-1 & ENST00000516594 & let-7b & ENST00000516461 \\
\hline mir-4454 & ENST00000459091 & let-7f-1 & ENST00000363271 & let-7b & ENST00000516775 \\
\hline mir-4454 & ENST00000466665 & let-7f-1 & ENST00000459091 & let-7b & ENST00000516594 \\
\hline ENST00000516572 & ENST00000459091 & let-7f-1 & ENST00000516350 & let-7b & ENST00000365160 \\
\hline ENST00000516351 & ENST00000459091 & let-7f-1 & ENST00000517209 & let-7b & ENST00000363271 \\
\hline ENST00000459091 & ENST00000463508 & let-7f-1 & ENST00000516501 & let-7b & ENST00000459091 \\
\hline
\end{tabular}


Table 4 Summary on combinations of two small RNAs (small RNA1 with small RNA2) which together completely discriminate metastasis subtypes (lymphogen, occult and their combination) from non-metastasized seminoma employing support vector machines (Continued)

\begin{tabular}{llllll}
\hline ENST00000459091 & ENST00000482884 & let-7f-1 & ENST00000516507 & let-7b & ENST00000516350 \\
ENST00000459091 & ENST00000488123 & let-7f-1 & ENST00000363865 & let-7b & ENST00000517209 \\
ENST00000517209 & ENST00000482884 & let-7f-1 & ENST00000362808 & let-7b & ENST00000516501 \\
\hline
\end{tabular}

From the total number of small RNA combinations per group (shown in parenthesis) only 87 combinations per group are presented.

nodes). Lymphogen metastasis and non-metastasized patients were matched with occult metastasized patients considering demographic and histological parameters where applicable as well as quality criteria for isolated RNA (Table 1). All patients included in this study were treated between 2008 and 2010 in one testis cancer centre. These 15 patients were selected out of about 300 seminoma patients, according to the criteria mentioned above.

\section{Histological examination}

All testicular tumours $(n=15)$ were examined by an experienced pathologist for histological and TNM classification (Table 1) and pure seminoma was diagnosed in all cases.

\section{Blood sample taking}

Whole blood samples $(2.5 \mathrm{ml})$ were taken intraoperative from cubital vein directly into the PAXgene Blood RNA system (BD Diagnostics, PreAnalytiX GmbH, Hombrechtikon, Switzerland). The tube was gently inverted (10 times), settled at room temperature overnight and stored at $-20^{\circ}$ until use. The Ethical Review Committee of the Medical Association Hamburg approved the study and all human samples were obtained with written informed consent.

\section{RNA Isolation}

After thawing the PAXGene tubes, and washing and centrifugation of the samples the cells became lysed (Proteinase K) followed by adding Lysis/Binding Solution taken from the mirVana Kit (Life Technologies, Darmstadt, Germany). With the mirVana kit total RNA including small RNA species was isolated by combining a Phenol-Chloroform RNA precipitation with further processing using a silica membrane. After several washing procedures DNA residuals became digested on the membrane (RNAse free DNAse Set, Qiagen, Hilden, Germany), washed, RNA was eluted in a collection tube and frozen at $-80^{\circ} \mathrm{C}$. Quality and quantity of isolated total RNA were measured spectrophotometrically (NanoDrop, PeqLab Biotechnology, Erlangen, Germany). RNA integrity was assessed by the 2100 Agilent Bioanalyser (Life Science Group, Penzberg, Germany) and DNA contamination was controlled by conventional PCR using actin primer.

For analysis, we used only RNA specimens with a ratio of A260/A280 $\geq 2.0$ (Nanodrop) and RNA integrity number
(RIN) between 6.0-9.3 for small RNA Next Generation Sequencing (IMGM Laboratories, Martinsried, Germany/ CeGat, Tübingen, Germany). For RNA samples with RIN below 7.0 or questionable $28 / 18 \mathrm{~S}$ bands (indications of RNA degradation) we performed additional checks to exclude the presence of RNAses and true RNA-degradation.

Small RNA next generation sequencing and data analysis We performed a genome wide small RNA sequencing using the SOLiD5500xl Next Generation Sequencing Technology (Life Technologies, Penzberg, Germany). In brief, total RNA was purified (PureLink microRNA isolation Kit), enriched small RNAs were ligated to SOLiD adaptors, reverse transcribed (SOLiD RT primer and ArrayScript RT), cDNA was purified (MinElute PCR purification Kit, Qiagen), a cut-off size of 60-80 nt was selected (Novex pre-cast gel products, invitrogen), cDNA was in-gel amplified and samples were barcoded using SOLiD 3'Barcode primer at the same time. Amplified cDNA was purified (PureLink PCR Micro Kit, Life Technologies) and used in emulsion PCR (SOLiD EZ Bead). Thereafter, the emulsion was broken to recover enriched beads and the so-called di-base probes were used by the SOLiD system in the sequencing-byligation procedure. Beside the SOLiD5500xl inherent software (LifeScope) used for image and signal processing, software CLC Genomics Workbench 5.1 (CLC bio) was used for clustering, counting, and annotation of all generated $75 \mathrm{bp}$ reads. After discarding reads without adaptor sequence and being shorter than $15 \mathrm{bp}$ (trimming) reads were assigned to known microRNAs (Sanger miRBase release 18, http://www.mirbase.org/) and known non-coding RNAs (ensembl database Homo sapiens.GRCh37.67.ncrna.fa, www.ensembl.org). Small RNAs showing a significant and $\geq 2$-fold differences in gene expression among groups and at least 50 reads were further analysed on their ability to separate both groups using univariate logistic regression analysis [21] and combinations of two small RNAs were examined employing support vector machines (linear Kermel).

\section{Abbreviations}

bp: Base pair; cDNA: Copy deoxyribonucleic acids; cS: clincal stage; CT scan: Computed tomography; DNA: Deoxyribonucleic acids; miRNA: micro ribonucleic acid; NGS: Next generation sequencing; qRT-PCR: Quantitative 
real time polymerase chain reaction; RNA: Ribonucleic acid; RIN: RNA integrity number; TNM-Classicfication: Tumor nodus metastasis classification.

\section{Competing interests}

The German Ministry of Defence supported this work. All authors declare that they have no competing interests.

\section{Authors' contributions}

CR has developed the study design, acquisition and interpretation of data and drafted the manuscript, DD has substantially contributed in the acquisition of data, MP and H-US have substantially contributed in the study design, interpretation of data and in revising the manuscript, WW and CM have made substantial contributions to acquisition of data and in revising the manuscript, BM-M has performed the statisitcal analysis of data and revised the manuscript, VM has made substantial contributions to technical procedures, data analysis and revised the manuscript, MA has developed the study design, supervised all technical/laboratory procedures and quality checks, analysis and interpretation of data and drafted the manuscript. All authors read and approved the final manuscript.

\section{Acknowledgements}

We would like to thank S. Senf for his skilful technical assistance. The German Ministry of Defence supported this work.

\section{Author details}

'Department of Urology, Federal Armed Forces Hospital, 22049 Hamburg, Germany. ${ }^{2}$ Bundeswehr Institute of Radiobiology, 80937 Munich, Germany. ${ }^{3}$ Department of Urology, Federal Armed Forces Central Hospital, 56072 Koblenz, Germany. ${ }^{4}$ Department of Hematology, Hemostasis Oncology and Stem Cell Transplantation, MHH, Hannover, Germany. ${ }^{5}$ Max Planck Institute of Psychiatry, Munich, Germany.

Received: 15 December 2013 Accepted: 24 February 2014 Published: 6 March 2014

\section{References}

1. Ruf CG, Isbarn H, Wagner W, Fisch M, Matthies C, Dieckmann KP: Changes in epidemiologic features of testicular germ cell cancer: age at diagnosis and relative frequency of seminoma are constantly and significantly increasing(). Urol Oncol 2014, 32(1):33. e1-6. doi:10.1016/j.urolonc.2012.12.002. Epub 2013 Feb 6.

2. Krege S, Beyer J, Souchon R, Albers P, Albrecht W, Algaba F, Bamberg M, Bodrogi I, Bokemeyer C, Cavallin-Stahl E, Classen J, Clemm C, Cohn-Cedermark G, Culine S, Daugaard G, De Mulder PH, De Santis M, de Wit M, de Wit R, Derigs HG, Dieckmann KP, Dieing A, Droz JP, Fenner M, Fizazi K, Flechon A, Fosså SD, del Muro XG, Gauler T, Geczi L, et al: European consensus conference on diagnosis and treatment of germ cell cancer: a report of the second meeting of the European Germ Cell Cancer Consensus group (EGCCCG): part I. Eur Urol 2008, 53:478-496.

3. Albers P, Albrecht W, Algaba F, Bokemeyer C, Cohn-Cedermark G, Fizazi K, Horwich A, Laguna MP: [EAU guidelines on testicular cancer: 2011 update. European Association of Urology]. Actas Urol Esp 2012, 36:127-145.

4. Ruf CG, Khalili-Harbi N, Sachs S, Isbarn H, Wagner W, Matthies C, Meineke V, Fisch M, Chun FK, Abend M: The search for biomarkers of metastatic seminoma. J Urol 2013, 190(3):1046-1051. doi:10.1016/j.juro.2013.04.022. Epub 2013 Apr 10

5. Kollmannsberger C, Tyldesley S, Moore C, Chi KN, Murray N, Daneshmand S, Black P, Duncan G, Hayes-Lattin B, Nichols C: Evolution in management of testicular seminoma: population-based outcomes with selective utilization of active therapies. Ann Oncol 2011, 22:808-814.

6. Dieckmann KP, Spiekermann M, Balks T, Flor I, Loning T, Bullerdiek J, Belge G: MicroRNAs miR-371-3 in serum as diagnostic tools in the management of testicular germ cell tumours. Br J Cancer 2012, 107:1754-1760.

7. Gillis AJ, Stoop HJ, Hersmus R, Oosterhuis JW, Sun Y, Chen C, Guenther S, Sherlock J, Veltman I, Baeten J, van der Spek PJ, de Alarcon P, Looijenga LH: High-throughput microRNAome analysis in human germ cell tumours. J Pathol 2007, 213:319-328.

8. Palmer RD, Murray MJ, Saini HK, van Dongen S, Abreu-Goodger C, Muralidhar B, Pett MR, Thornton CM, Nicholson JC, Enright AJ, Coleman N, Children's Cancer and Leukaemia Group: Malignant germ cell tumors display common
microRNA profiles resulting in global changes in expression of messenger RNA targets. Cancer Res 2010, 70:2911-2923.

9. Port M, Glaesener S, Ruf C, Riecke A, Bokemeyer C, Meineke V, Honecker F, Abend M: Micro-RNA expression in cisplatin resistant germ cell tumor cell lines. Mol Cancer 2011, 10:52.

10. Liu X, Yu J, Jiang L, Wang A, Shi F, Ye H, Zhou X: MicroRNA-222 regulates cell invasion by targeting matrix metalloproteinase 1 (MMP1) and manganese superoxide dismutase 2 (SOD2) in tongue squamous cell carcinoma cell lines. Cancer Genomics Proteomics 2009, 6:131-139.

11. Profumo V, Gandellini P: MicroRNAs: cobblestones on the road to cancer metastasis. Crit Rev Oncog 2013, 18:341-355.

12. Ruf CG, Nastaly P, Becker P, Isbarn H, Honecker F, Pantel K, Riethdorf S, Hoeppner D, Fisch M, Wagner W, Ahyai S: Circulating tumor cells can be detected in patients with testicular germ cell tumors. J Urol 2013, 189:e289.

13. Mayr C, Hemann MT, Bartel DP: Disrupting the pairing between let-7 and Hmga2 enhances oncogenic transformation. Science 2007, 315:1576-1579.

14. Johnson SM, Grosshans H, Shingara J, Byrom M, Jarvis R, Cheng A, Labourier E, Reinert KL, Brown D, Slack FJ: RAS is regulated by the let-7 microRNA family. Cell 2005, 120:635-647.

15. Niopoulos D, Hirsch HA, Struhl K: An epigenetic switch involving NF-kappaB, Lin28, Let-7 MicroRNA, and IL6 links inflammation to cell transformation. Cell 2009, 139:693-706.

16. Musumeci M, Coppola V, Addario A, Patrizii M, Maugeri-Sacca M, Memeo L, Colarossi C, Francescangeli F, Biffoni M, Collura D, Giacobbe A, D'Urso L, Falchi M, Venneri MA, Muto G, De Maria R, Bonci D: Control of tumor and microenvironment cross-talk by miR-15a and miR-16 in prostate cancer. Oncogene 2011, 30:4231-4242.

17. Yu Z, Willmarth NE, Zhou J, Katiyar S, Wang M, Liu Y, McCue PA, Quong AA, Lisanti MP, Pestell RG: microRNA 17/20 inhibits cellular invasion and tumor metastasis in breast cancer by heterotypic signaling. Proc Natl Acad Sci U S A 2010, 107:8231-8236.

18. Ruf CG, Linbecker M, Port M, Riecke A, Schmelz HU, Wagner W, Meineke V, Abend $\mathrm{M}$ : Predicting metastasized seminoma using gene expression. BJU Int 2012, 110:E14-E20.

19. Port M, Wang Y, Schmelz HU, Pottek T, Meineke V, Ruf C, Abend M: A gene signature of primary tumor identifies metastasized seminoma. Urol Oncol 2011, 29:764-773.

20. Port M, Seidl C, Ruf CG, Riecke A, Meineke V, Abend M: Reliable and sample saving gene expression analysis approach for diagnostic tool development. Health Phys 2012, 103:159-168.

21. Baggerly KA, Deng L, Morris JS, Aldaz CM: Overdispersed logistic regression for SAGE: modelling multiple groups and covariates. BMC Bioinform 2004, 5:144.

\section{doi:10.1186/1476-4598-13-47}

Cite this article as: Ruf et al: Small RNAs in the peripheral blood discriminate metastasized from non-metastasized seminoma. Molecular Cancer 2014 13:47.

\section{Submit your next manuscript to BioMed Central and take full advantage of:}

- Convenient online submission

- Thorough peer review

- No space constraints or color figure charges

- Immediate publication on acceptance

- Inclusion in PubMed, CAS, Scopus and Google Scholar

- Research which is freely available for redistribution 